\title{
Fracture of Pelvis
}

National Cancer Institute

\section{Source}

National Cancer Institute. Fracture of Pelvis. NCI Thesaurus. Code C35153.

A traumatic break in one or more of the bones that compose the bony structure of the pelvis. 